\title{
A Toolbox for
}

\section{Effleient Parameter and Structure Variation of Time Domain Power System Simulation Models in Simulink}

\section{Paper ID 498348}

Tilman Wippenbeck Graduate Student Member IEEE, Reinhold Bertram, Armin Schnettler Member IEEE Institute for High Voltage Technology, RWTH Aachen University, Aachen, Germany

stoical@ifht.rwth-aachen.de

\section{Introduction}

Use-Case: Model parameter \& structure variation of Simulink models

\section{Addressed scenarios:}

complex / large / "growing" Simulink models

multiple research tasks

- collaboration of researchers on one model

flexible uniform, group-wise or individual settings specific to task

automation of a large number of experiments

Aim: focus on research (modelling, variation studies \& analyses) rather than implementation of required tools
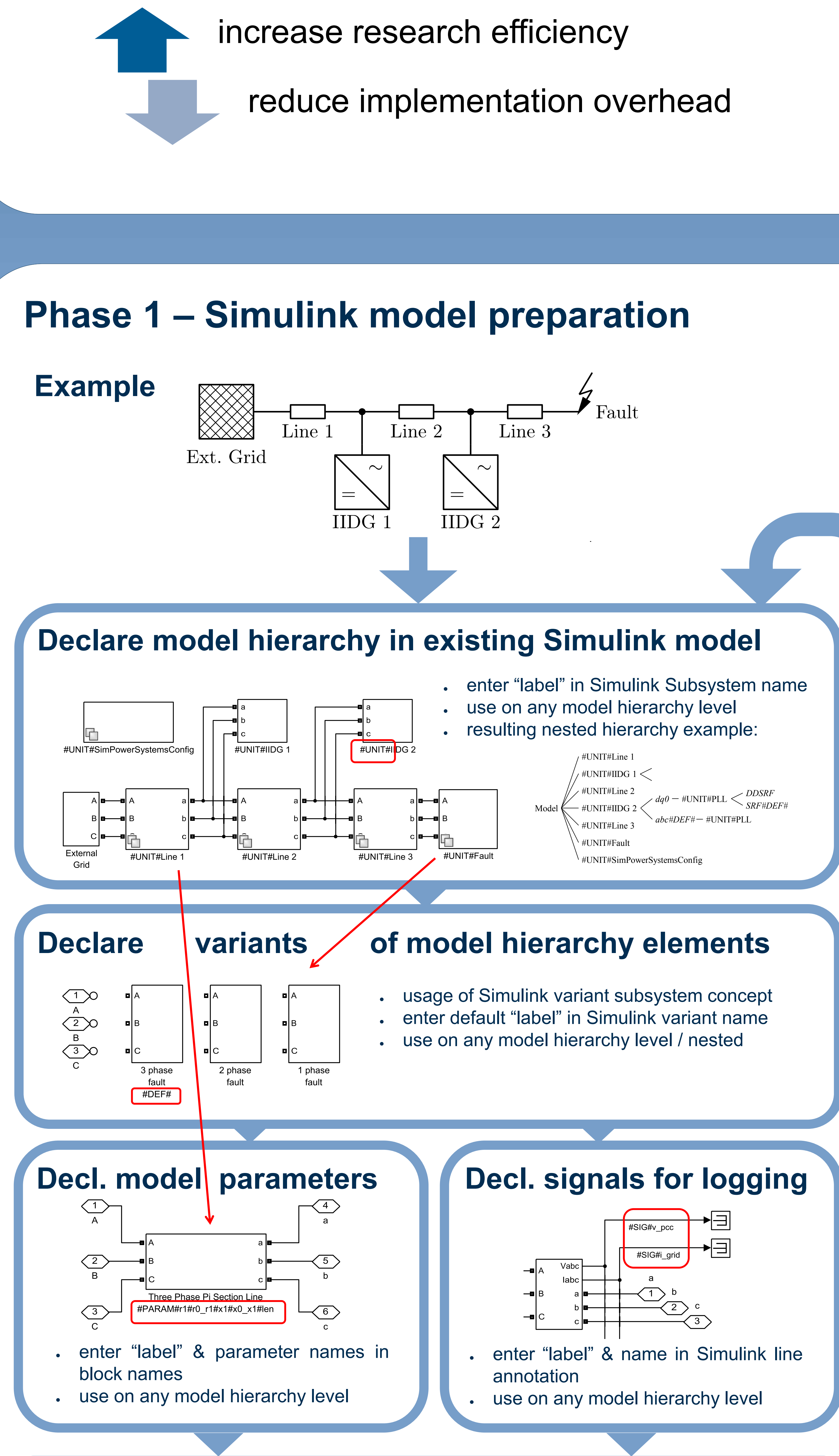

Register elements to Toolbox (script)

Use model

\section{major model changes:} extend as necessary

\section{Phase 2 - Simulink model usage}

Principle

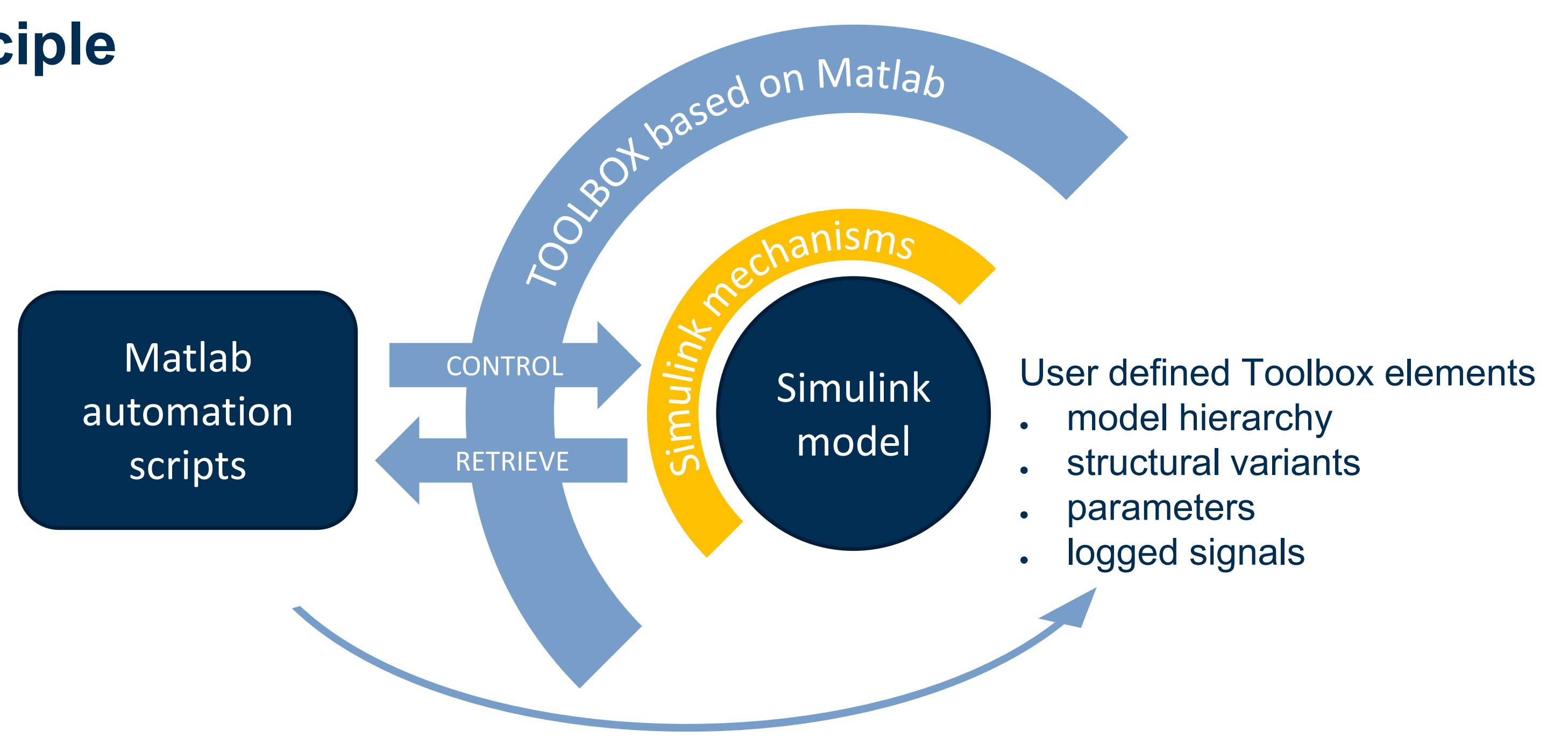

a "labelpath" efficiently addresses elements - address individually, e.g. 'HLine 1\#r1' exploiting Toolbox hierarchy \& element names : address groups, e.g.

Process description

elements map to supentor herarchy element

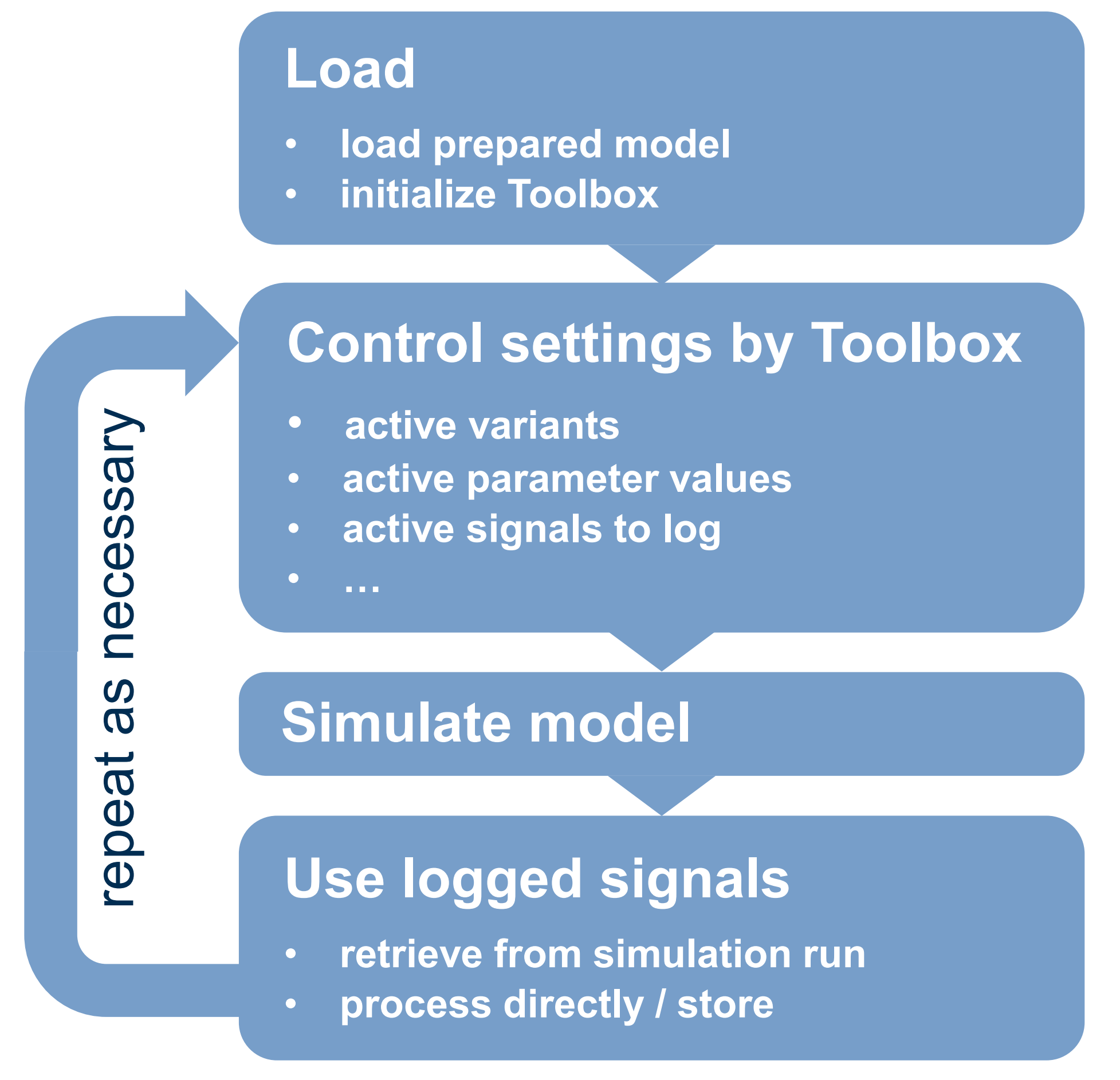

quick initialization routines \& example scripts provided

routines \& example scripts provided exploit defined model hierarchy in addressing element

strategies for minimizing accelerato recompiles

routines \& example scripts provided usage of best practice approaches for large numbers of experiments database connectivity

\section{Phase 3 - Analysis of experiments}

- retrieve logged signals from storage by labelpath for further processing routines \& example scripts provided

\section{Conclusions}

Increase of research efficiency:

- quick definition of parameters, structural variants and signals to log by means of labels in Simulink block names or connection lines

meaningful syntax for addressing elements incl. regular expressions

- unified processes for automated variation studies \& result access

Applicability to other research areas

generic approach and mechanisms

usability with most Simulink toolboxes

\section{Access}

The authors intend to publish the Toolbox source code and documentation as free and open source software. Please visit: www.ifht.rwth-aachen.de/en/stoical 